\title{
Analytic free-form lens design for tracking integration in concentrating photovoltaics
}

\author{
Fabian Duerr, ${ }^{*}, a$ Pablo Benítez, ${ }^{b, c}$ Juan Carlos Miñano, ${ }^{b, c}$ Youri Meuret ${ }^{a}$ and Hugo \\ Thienpont ${ }^{a}$ \\ ${ }^{a}$ Vrije Universiteit Brussel, Faculty of Engineering Sciences, Brussels Photonics Team \\ B-PHOT, TONA-FirW, Pleinlaan 2, 1050 Brussels, Belgium \\ ${ }^{b}$ CeDInt, Universidad Politécnica de Madrid (UPM), Campus de Montegancedo, 28223 \\ Pozuelo de Alarcón, Spain \\ ${ }^{c}$ LPI, 16662 Hale Ave., Irvine, CA USA 92606
}

\begin{abstract}
In this work the concept of tracking integration in concentrating photovoltaics (CPV) is revisited and developed further. With respect to conventional CPV, tracking integration eliminates the clear separation between stationary units of optics and solar cells, and external solar trackers. This approach is capable of further increasing the concentration ratio and makes high concentrating photovoltaics $(>500 \times)$ available for single-axis tracker installations. The reduced external solar tracking effort enables possibly cheaper and more compact installations. Our proposed optical system uses two laterally moving plano-convex lenses to achieve high concentration over a wide angular range of $\pm 24^{\circ}$. The lateral movement allows to combine both steering and concentration of the incident direct sun light. Given the specific symmetry conditions of the underlying optical design problem, rotational symmetric lenses are not ideal for this application. For this type of design problems, a new free-form optics design method presented in previous papers perfectly matches the symmetry. It is derived directly from Fermat's principle, leading to sets of functional differential equations allowing the successive calculation of the Taylor series coefficients of each implicit surface function up to very high orders. For optical systems designed for wide field of view and with clearly separated optical surfaces, this new analytic design method has potential application in both fields of nonimaging and imaging optics.
\end{abstract}

Keywords: Solar concentrators, Geometric optical design, Mathematical methods in physics, Free-form optics

\section{INTRODUCTION}

Concentrating photovoltaic (CPV) systems employ optics to concentrate direct sunlight onto small solar cells. The significant decrease of the required solar cell area provides a pathway to lower cost, as expensive semiconductor material is replaced with inexpensive mirrors or lenses by a factor roughly equal to the concentration factor. Furthermore, high-efficiency multi-junction (MJ) solar cells made by III-V elements of the period table can be used to boost the conversion efficiency of CPV modules beyond 30\%, making CPV the most efficient among the PV technologies. To be economically viable, the use of expensive MJ solar cells needs high concentration - typically exceeding 400 suns. This level of concentration normally demands an accurate dual-axis tracking of the sun's diurnal and seasonal movement. Most of today's CPV manufacturers in the high-concentration range tend to work with very large modules and pedestal-mounted dual-axis trackers as illustrated by three examples in Fig. 1. The three shown CPV manufacturers Amonix, Soitec (which recently acquired Concentrix Solar), and Solfocus currently lead the race at an utility-scale.

Well-suited for utility-scale power plants, these installations are less adequate for small to mid-scale solutions. Single-axis trackers, on the other hand, are already in use on flat rooftops for photovoltaic (PV) modules. However, CPV systems designed for single-axis trackers are limited in the achievable level of concentration to about $300 \times$ for polar alignment, where the tracker axis equals the earth's axis of rotation. ${ }^{1}$ This value is not high enough to make economic use of MJ solar cells.

\footnotetext{
*E-mail correspondence: fduerr@b-phot.org; Telephone: +32 (0)2 6293570
} 


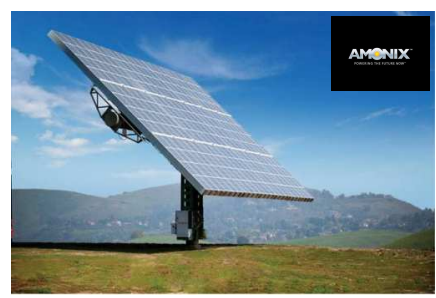

(a)

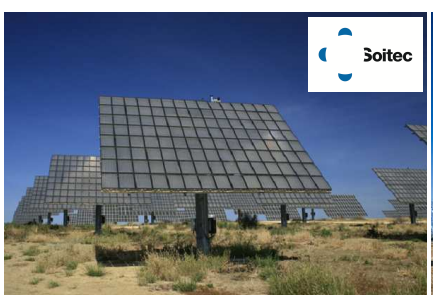

(b)

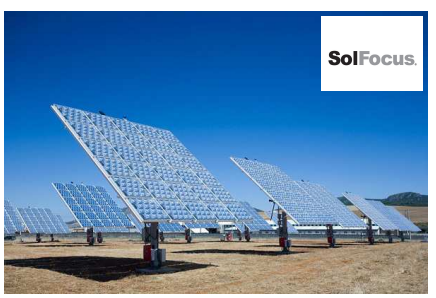

(c)

Figure 1. Exemplary CPV installations of (a) Amonix, (b) Soitec Concentrix and (c) SolFocus. Photo courtesy of (a) Jeff Aubin, (b) Concentrix and (c) SolFocus

Despite the vast number of differing CPV system designs, almost all have something essential in common: the CPV modules - consisting of stationary concentrating optics plus deployed solar cells - and the external trackers are treated separately. In contrast to this clear separation, the concept of tracking integration embeds part of the external solar tracking functionality in the CPV module. ${ }^{2}$ This offers a strong reduction of the external solar tracking effort whilst maintaining high concentration levels.

This article is structured as follows. The general concept of tracking integration in concentrating photovoltaics is discussed in Section 2. In Section 3, we present our tracking integration approach using laterally moving optics. The analysis of this optical design is presented in Section 4, where steps towards a tailored free-form solution are elaborated. Finally, in Section 5, conclusions are drawn and an outlook is given

\section{TRACKING INTEGRATION IN CONCENTRATING PHOTOVOLTAICS}

The concept of tracking integration in CPV is enjoying more and more attention. Very different optical designs following this approach have been discussed in literature, and first start-up companies underline the level of interest in this new concept. In general terms, it is possible to distinguish between two specific application types.

1. Reduce the external tracking effort in favor of a compact installation size (see, for example ${ }^{3-6}$ )

2. Fine-tune the tracking functionality allowing coarse external solar tracking ${ }^{7}$

Furthermore, it is of course possible to combine both functionalities to reduce the external tracking effort and to allow coarse remaining external solar tracking at the same time. To do so, it is essential to introduce additional degrees of freedom to the CPV module, such as the relative movement between optics and solar cells. Relative movements can be achieved through rotary motions or rectilinear motions as it is illustrated in Fig. 2(a) and (b). Tracking integration concepts based on rotary motions are used, for example, by companies SunCycle ${ }^{8}$ and zettasun. ${ }^{9}$ A tracking integration approach based on rectilinear motions is used by Sunfish Solar. ${ }^{10}$ Fig. 2(c) shows a motion-free alternative. The tracking integration is based on liquid crystal (LC) light steering for line concentration.

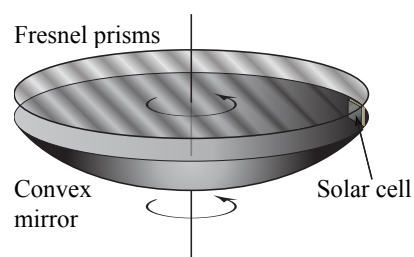

(a)

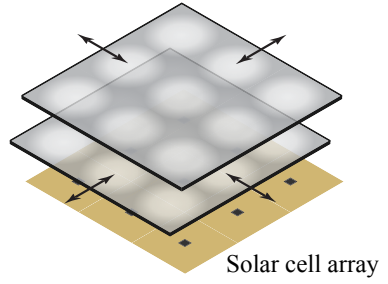

(b)

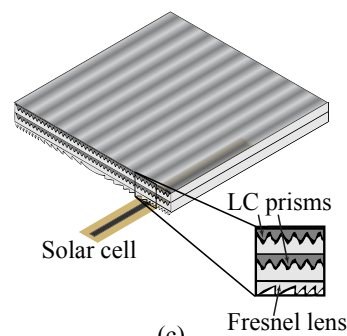

(c)

Figure 2. Different tracking integration concepts: using (a) rotary or (b) rectilinear motions; a motion-free example (c) based on liquid crystal (LC) light steering 
Depending on the application type and the chosen optical design for tracking integration, external solar tracking might still be needed. A clear drawback of reducing the external solar tracking is the reduced yearly insolation (incoming solar radiation) due to off-axis cosine losses, especially in case of statically mounted solar panels. For dual-axis trackers, as illustrated in Fig. 3(a), the yearly insolation is maximal as the modules always point at the sun. However, the comparison of the potential annual energy yield for polar aligned single-axis tracker with dual-axis tracker installations shows moderate differences, e.g. for miscellaneous places in Europe. ${ }^{11}$

Fig. 3(b) shows such a polar aligned single-axis tracker where the tracker axis equals the earth's axis of rotation. For horizontal single-axis trackers, as shown in Fig. 3(c), the yearly insolation is typically lower.

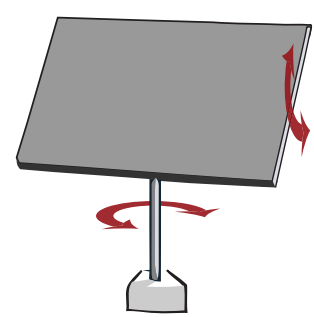

(a)

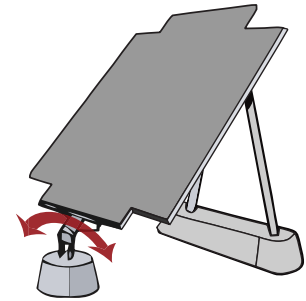

(b)

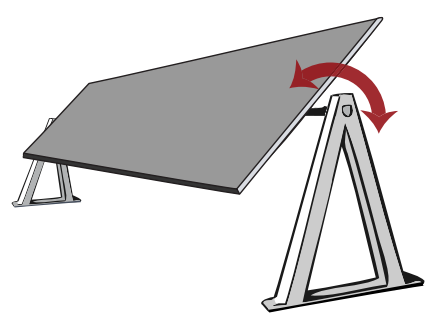

(c)

Figure 3. Different solar trackers used in both PV and CPV; (a) dual-axis tracker, (b) polar aligned single-axis tracker, and (c) horizontal single-axis tracker

It becomes evident that the application of dual-axis trackers in conventional high concentrating photovoltaics mainly arises from the theoretical point concentration limit for single-axis trackers rather than a maximized insolation. Tracking integration allows to weaken or even break this limitation.

\section{TRACKING INTEGRATION USING LATERALLY MOVING OPTICS}

In our approach, tracking integration is used to further increase the point concentration ratio for polar aligned single-axis tracker installations. The polar alignment offers a strong reduction of the external solar tracking effort in comparison to dual-axis tracking whilst maintaining high insolation levels. The laterally moving optics arrays combine both concentration and steering of the incident direct sun light. Fig. 4 illustrates the inherent difference between conventional CPV and tracking integration. Fig. 4(a) shows a conventional CPV module consisting of stationary concentrating optics plus deployed solar cells - and the external tracker. Fig. 4(b) shows the schematic assembly of our module.

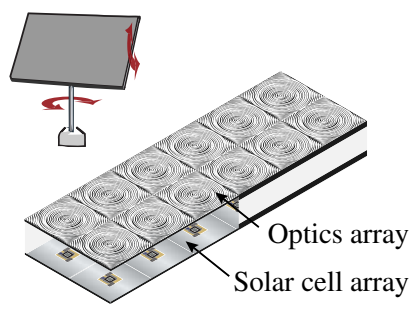

(a)

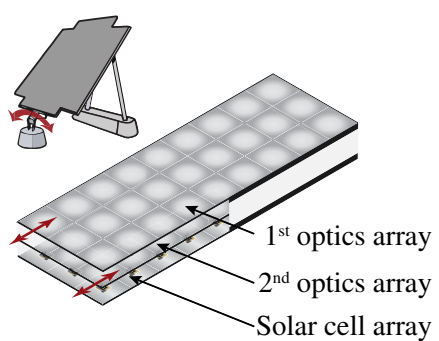

(b)

Figure 4. Schematic assembly of (a) conventional CPV module for pedestal-mounted dual-axis trackers and (b) our tracking integrated CPV module for polar aligned single-axis trackers

The polar aligned single-axis tracker is installed towards the South. In this particular configuration, the tilt angle is equal to the latitude of the installation site and the rotational axis of the single axis tracker equals the earth's axis of rotation. The aperture angle which has to be covered by the tracking integration equals $\pm 24^{\circ}$ (axial tilt of the earth) in North-South direction and the sun's half divergence angle in East-West direction. Furthermore, additional lateral shifts in East-West direction could be used to fine-tune the tracking functionality lowering the demands on the applied external single-axis tracking installation. 


\section{STEPS TOWARDS A TAILORED FREE-FORM SOLUTION}

Regarding the optics design of our system, the tracking integration has to cover an angular range of $\pm 24^{\circ}$ for incident direction vectors (pointing at the sun) within a single plane. Symmetry considerations make evident that rotational symmetric lenses are not an optimal solution. Therefore, we developed a new design algorithm, ${ }^{12}$ based on the Simultaneous Multiple Surface (SMS) design method in three dimensions. ${ }^{13}$ It allows the simultaneous calculation of two optical surfaces focusing two off-axis ray sets. Fig. 5(a) shows the obtained plano-convex free-form lenses with rectangular apertures. Fig. 5(b) shows obtained ray simulation results demonstrating that

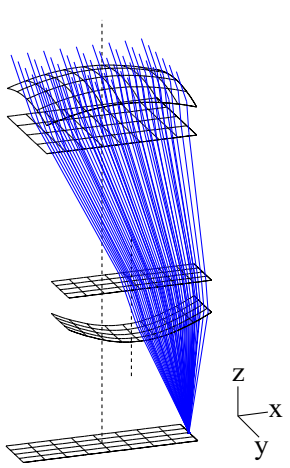

(a)

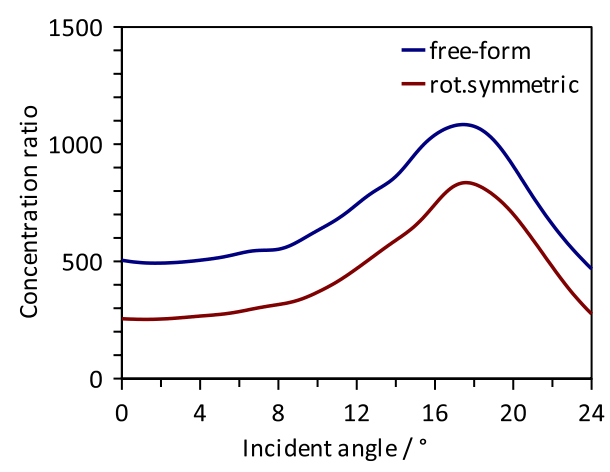

(b)

Figure 5. (a) Laterally moving free-form lens design based on SMS3D; (b) Concentration ratio against incident angle for free-form (upper curve) and rotational symmetric lens design (lower curve) for rectangular lens and receiver apertures

this free-form lens design outperforms its rotational symmetric counterpart. Clearly exceeding the desired $400 \times$ concentration on a polar aligned single-axis tracker over the complete field of view.

This optical design has two distinct features: its wide field of view and a clear separation between the two optical surfaces. Fig. 6(a) shows a simplified SMS2D reference design sharing identical characteristics.

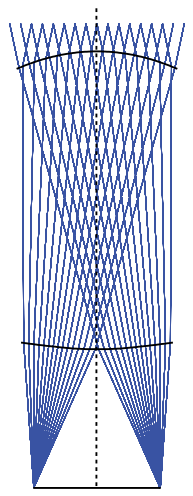

(a)

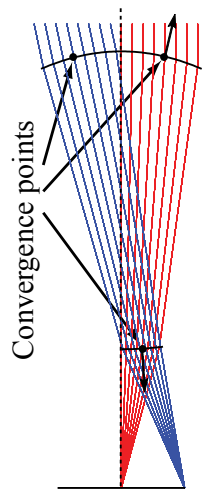

(b)

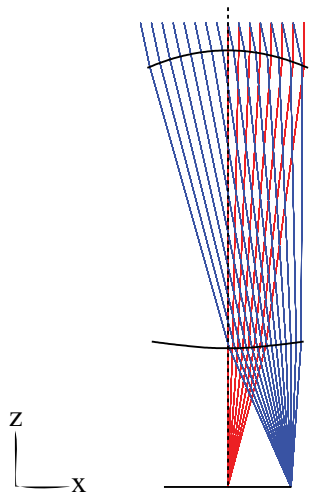

(c)

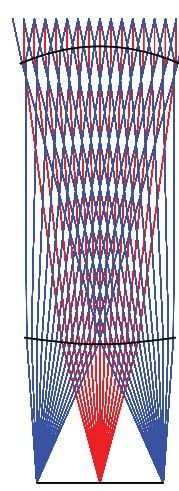

(d)

Figure 6. Steps (a)-(c) show how to couple an additional on-axis ray set starting from a simplified SMS2D reference design; illustration $(\mathrm{d})$ shows the final ray paths

We have demonstrated with a new analytic design algorithm that it is possible to couple more than two ray sets with only two lens surfaces for such configurations. ${ }^{14}$ This can only be achieved if different ray sets use different portions of the lens surfaces. Key to this new design method are the convergence points, which are characterized by on- and off-axis rays sharing identical points and normal vectors on each lens profile as shown in Figure 4(b). Convergence points were first introduced to design aspheric V-groove reflectors. ${ }^{15,16}$ Fermat's principle was used to deduce a set of functional differential equations fully describing the entire optical system. The derived general analytic solution makes it possible to calculate the lens profiles up to $20^{\text {th }}$ order Taylor series about the convergence points. ${ }^{17}$ 
In a next step, we generalized the two-dimensional analytic optics design method to the three-dimensional case. ${ }^{18}$ In a similar way, Fermat's principle is used to derive additional sets of functional differential equations which makes it possible to calculate the Taylor series functions describing the free-form lens surfaces by more than 100 coefficients. Fig. 7 shows two exemplary calculated solutions, a meniscus (a) and a biconvex (b) lens. We already

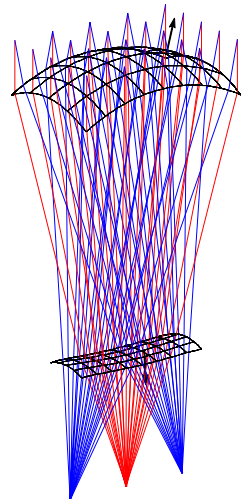

(a)

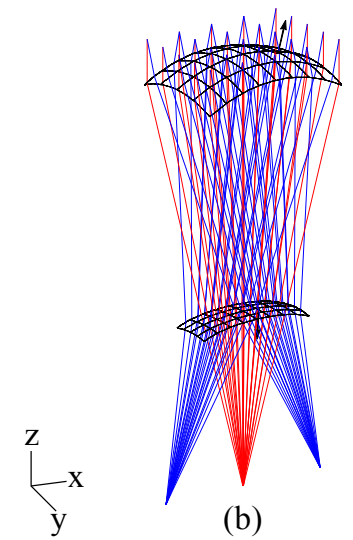

(b)

Figure 7. Ray tracing results for two exemplary calculated free-form lenses, a meniscus (a) and a biconvex (b) lens

demonstrated the potential use of this new free-form optics design method for imaging applications with a high aspect ratio. ${ }^{19}$ Finally, to incorporate the laterally movement of the lenses, two extensions are needed in the analytic design process. First, the single thick lens has to be divided into two plano-convex lenses as it is shown in Fig. 5(a). The lateral movement will be implemented by shifting the lower lens for off-axis design angles, very similar to the extended SMS3D design algorithm. ${ }^{12}$

A further extension could be the case in which the movement is no longer lateral but follows a curved trajectory. In the case of beam-steering array optics designs with the SMS method, it has been shown recently that curved tracking trajectory helps to broaden the incident angle's range significantly. ${ }^{20}$ In addition, further desired attributes of concentrating photovoltaic systems are the uniformity of the flux, color mixing on the solar cell's surface and an increased acceptance angle which is directly related to misalignment tolerance. A well accepted solution to achieve this is a final stage concentrator on top of the solar cell. Future work will also cover this component and help to increase the aperture angle, ensure color mixing and increase the acceptance angle. A final polychromatic optimization will then help to tap the full potential of this tracking integration concept.

\section{CONCLUSIONS}

Within the scope of this work, we have presented the concept of tracking integration in concentrating photovoltaics and indicated its potential to open small to mid-scale installation markets for CPV due to the strong reduction of the external solar tracking effort. Our new free-form design method shows a way to unleash the full potential of the considered optical system. Future work intends to apply the analytic design method to the presented tracking integration approach using laterally moving optics and work on a first demonstrator.

\section{ACKNOWLEDGMENTS}

Work reported in this paper is supported in part by the Research Foundation - Flanders (FWO Vlaanderen) that provides a PhD grant (grant number FWOTM510) and provided a grant for a research period at CeDInt, Universidad Politécnica de Madrid (grant number V424711N) for Fabian Duerr, and in part by the IAP BELSPO VI-10 under grant IAP P7-35 photonics@be, the Industrial Research Funding (IOF), Methusalem, and the OZR of the Vrije Universiteit Brussel.

Work is also supported by projects TEC2011-24019 and SEM: TSI-020302-2010-65, funded by the Spanish Ministries MEC and MITYC, respectively. 


\section{REFERENCES}

[1] Mohr, A., Roth, T., and Glunz, S., "BICON: high concentration PV using one-axis tracking and silicon concentrator cells," Prog. Photovoltaics Res. Appl. 14(7), 663-674 (2006).

[2] Duerr, F., Meuret, Y., and Thienpont, H., "Tracking integration in concentrating photovoltaics using laterally moving optics," Opt. Express 19(103), A207-A218 (2011).

[3] Karp, J., Tremblay, E., and Ford, J., "Planar micro-optic solar concentrator," Opt. Express 18(2), 1122-1133 (2010).

[4] Hallas, J., Karp, J., Tremblay, E., and Ford, J., "Lateral translation micro-tracking of planar micro-optic solar concentrator," Proc. SPIE 7769, 776904 (2010).

[5] Baker, K., Karp, J., Tremblay, E., Hallas, J., and Ford, J., "Reactive self-tracking solar concentrators: concept, design, and initial materials characterization," Appl. Opt. 51(8), 1086-1094 (2012).

[6] Hallas, J., Baker, K., Karp, J., Tremblay, E., and Ford, J., "Two-axis solar tracking accomplished through small lateral translations," Appl. Opt. 51(25), 6117-6124 (2012).

[7] Sweatt, W., Jared, B., Nielson, G., Okandan, M., Filatov, A., Sinclair, M., Cruz-Campa, J., and Lentine, A., "Micro-optics for high-efficiency optical performance and simplified tracking for concentrated photovoltaics (CPV)," Proc. SPIE 7652, 765210 (2010).

[8] SunCycle. Retrieved Oct. 25, 2012, from http://www.suncycle.nl/

[9] Zettasun, Inc.. Retrieved Oct. 25, 2012, from http://www.zettasun.com/zettasun/technology.html

[10] Sunfish Energy Ltd. Retrieved Oct. 25, 2012, from http://www.sunfishsolar.com/

[11] Huld, T., Cebecauer, T., Súri, M., and Dunlop, E., "Analysis of one-axis tracking strategies for PV systems in Europe," Prog. Photovoltaics Res. Appl. 18(3), 183-194 (2010).

[12] Duerr, F., Benítez, P., Miñano, J., Meuret, Y., and Thienpont, H., "Integrating tracking in concentrating photovoltaics using non-rotational symmetric laterally moving optics," Proc. SPIE 8124, 81240M (2011).

[13] Benítez, P., Miñano, J., Blen, J., Mohedano, R., Chaves, J., Dross, O., Hernandez, M., and Falicoff, W., "Simultaneous multiple surface optical design method in three dimensions," Opt. Eng. 43, 1489 (2004).

[14] Duerr, F., Benítez, P., Miñano, J. C., Meuret, Y., and Thienpont, H., "Analytic design method for optimal imaging: coupling three ray sets using two free-form lens profiles," Opt. Express 20(5), 5576-5585 (2012).

[15] Grabovičkić, D., Benítez, P., and Miñano, J., "Aspheric V-groove reflector design with the SMS method in two dimensions," Opt. Express 18(3), 2515-2521 (2010).

[16] Grabovičkić, D., Benítez, P., and Miñano, J., "Free-form V-groove reflector design with the SMS method in three dimensions," Opt. Express 19(104), A747-A756 (2011).

[17] Duerr, F., Benítez, P., Miñano, J. C., Meuret, Y., and Thienpont, H., "Perfect imaging of three object points with only two analytic lens surfaces in two dimensions," Proc. SPIE 8429, 842908 (2012).

[18] Duerr, F., Benítez, P., Miñano, J. C., Meuret, Y., and Thienpont, H., "Analytic free-form lens design in 3D: coupling three ray sets using two lens surfaces," Opt. Express 20(10), 10839-10846 (2012).

[19] Duerr, F., Benítez, P., Miñano, J. C., Meuret, Y., and Thienpont, H., "Analytic free-form lens design for imaging applications with high aspect ratio," Proc. SPIE 8486, 848609 (2012).

[20] Lin, W., Benítez, P., and Miñano, J. C., "Beam-steering array optics designs with the SMS method," Proc. SPIE 8485, 848505 (2012). 